\title{
III-V on Si components for packet switching
}

\author{
G. Morthier*, M. Tassaert*, P. Méchet*, O. Raz**, H. Dorren**, D. Van Thourhout* and G. Roelkens* \\ * Photonics Research Group, Department of Information Technology, Ghent University-imec, Sint-Pietersnieuwstraat 41, \\ B-9000 Ghent, Belgium \\ ** Eindhoven University of Technology, Den Dolech 2, 5600MB Eindhoven, The Netherlands
}

\begin{abstract}
We discuss the use of active and passive InP membrane structures, heterogeneously integrated onto SOI passive circuits, for switching applications such as gating, wavelength conversion and all-optical flip-flopping. Devices include microdisk lasers and resonators, as well as travelling wave structures, in either electrically pumped or unpumped configuration. We also pay some attention to the fabrication aspects.
\end{abstract}

\section{INTRODUCTION}

All-optical networks would benefit greatly from an increased use of all-optical signal processing, especially with the introduction of all-optical packet/burst switching [1]. In addition to wavelength conversion and signal regeneration, photonic devices implementing digital or logic functions are greatly desired, e.g. for header processing, routing or storing header information.

Many components that could be of use in optical switching have been demonstrated using an all-InP approach [2]. However, many of these solutions require rather complex integrated circuits, which are difficult to fabricate reliably in InP or which contain several powerhungry semiconductor optical amplifiers. The low index contrast of InP waveguides also leads to a rather large footprint of these PICs.

InP membranes, with thicknesses of $500 \mathrm{~nm}$ or less, as well as the SOI passive circuits on which they are integrated heterogeneously provide a much better optical confinement. This allows the realization of components and PICs with significantly smaller dimensions, but also with lower required optical power levels and/or lower electrical power consumption. Moreover, the possibility to fabricate the SOI passive circuits using mature CMOS fabrication tools makes this approach very suited for the realization of PICs comprised of complex SOI passive circuits and a limited number of active (InP) devices.

Below we will give more details on the fabrication technology, discuss some interesting devices that have been realized in it and conclude with some ideas for further improvement and expansion of the technology.

\section{BONDING AND INTEGRATION TECHNOLOGY}

The heterogeneous integration is achieved by bonding an InP die (or wafer) to an SOI die (or wafer), either using molecular or adhesive bonding (using a BCB polymer). Adhesive bonding has some advantages such as relaxed requirements on surface cleanliness and roughness. By depositing an oxide (alumina or silica) on the InP die prior to bonding, extremely high bonding yields can be achieved. Combining the oxide, of which the thickness can be controlled accurately, with a very thin BCB layer furthermore allows a good control of the total bonding layer thickness. Very high bonding yields, as well as the possibility of very thin bonding layers, have recently also been demonstrated using a commercial wafer bonder [3].

After bonding, the InP dies can be processed using identical processes as those used in the fabrication of full InP devices. Using e-beam or deep-UV lithography, the InP waveguides can be very accurately aligned with respect to the underlying silicon wire waveguides, and the dimensions of microdisks or straight InP waveguides can be well controlled.

\section{MICRODISK LASERS AND RESONATORS}

Microdisk lasers were among the first laser diodes demonstrated using bonding of InP on SOI [4]. With diameters of $7.5 \mu \mathrm{m}$ and threshold currents below $0.5 \mathrm{~mA}$, they are among the heterogeneously integrated and electrically pumped laser diodes with the smallest footprint and power consumption.

In recent years, such microdisk lasers have been demonstrated in a number of switching applications. If the sidewall roughness and external reflections (from facets or grating couplers) are sufficiently small, such lasers can operate in a unidirectional mode. Switching between both a clock and counter clock wise mode can be done all-optically, thus allowing the implementation of all-optical flip-flops. In [5], record low switching energies of $1.8 \mathrm{fJ}$ were reported, in addition to switching times of 60 ps and low power consumption.

Unbiased microdisk resonators were later used also for applications such as gating, wavelength conversion and even format conversion, with speeds up to $10 \mathrm{Gbps}$. In this case, however, the wavelengths of incoming and converted/gated signal are fixed to the resonances of the microdisks. Typical required switching pulses have an energy of $150 \mathrm{fJ}$ (in the silicon waveguide).

As microdisk resonators have also been shown to operate as resonant detectors and modulators [6], it is in principle possible to fabricate very functional PICs using multiple microdisks, mutually interconnected via $\mathrm{Si}$ wires. This requires an optimized processing technology, guaranteeing that the lasing and/or resonant wavelengths of nominally identical microdisks on a chip are as close 
as possible to each other. Although such reproducibility can theoretically be achieved using advanced lithography, perhaps in combination with thermal tuning, there are alternatives which are wavelength independent.

\section{INP MEMBRANE TRAVELLING WAVE SAS}

A recently reported wavelength independent optical gate is the travelling wave InP membrane switch shown in Figure 1 [7]. The membrane acts as saturable absorber (SA) with very small dimensions (InP layer thickness of $100 \mathrm{~nm}$ ) and high optical confinement. Coupling between the silicon and the InP waveguide is maximized by the use of inverted tapers in the silicon and adiabatic tapers in the InP. The active structure consists of 3 quantum wells and thanks to the high index contrast between InP and BCB a confinement of $17 \%$ is obtained in these QWs. A rib waveguide structure is used because the shallow etched cladding layers create extra heat sinking paths.

When used as optical switch or gate, the transmission of the waveguide is switched by optical absorption of a control (pump) beam. For a pump power of $4.5 \mathrm{dBm}$, an extinction ratio of over $30 \mathrm{~dB}$ over the entire $\mathrm{C}$ band is found for a $1500 \mathrm{~nm}$ pump in a device of $150 \mu \mathrm{m}$ long [7].

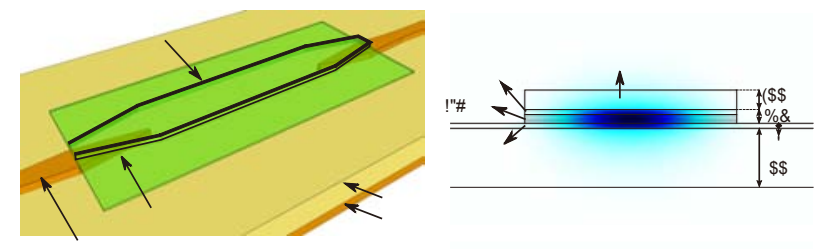

Fig. 1. (a) Schematic view of the thin travelling wave membrane switch, (b) Mode profile in the III-V membrane waveguide and cross section of the intensity in the center of the waveguide.

First dynamic measurements with this type of gate indicate that the maximum bitrate is limited to a few Gbps. This is due to the rather large carrier lifetime. Work is in progress to reduce the carrier lifetime and increase the maximum speed, e.g. by ion implantation or increasing the surface recombination.

The device has also been operated as a passive signal regenerator, giving extinction ratio improvement for low input extinction ratios. For a $-1.5 \mathrm{dBm} 1 \mathrm{Gbps}$ input signal at $1530 \mathrm{~nm}$, the ER improved from 2 to $6.2 \mathrm{~dB}$, leading to an anticipated negative power penalty of $4.5 \mathrm{~dB}$. Figure 2(a) shows the obtained ER and the insertion loss vs. the input power, while Figure 2(b) shows the measured BER vs. the average received power at $1 \mathrm{Gbps}$.
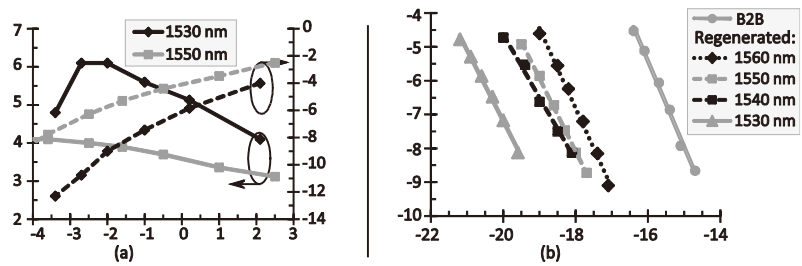

Fig. 2. (a) Output ER and insertion loss vs. input power. (b) BER vs. average received power at $1 \mathrm{Gbps}$.

\section{CONCLUSIONS AND OUTLOOK}

In this paper we have illustrated several heterogeneously integrated III-V on Si devices with low power consumption or operating with lower optical input powers. Integrating a few of such individual components together on a single SOI circuit makes it possible to realize PICs with more functionality. E.g. a microdisk alloptical set-reset flip-flop can be integrated with a membrane TW-SA to obtain a D-flip-flop.

Other key devices for optical packet switching which should be implemented in the future using the heterogeneous integration approach may include fast (electronically) tunable lasers. Work has also started to investigate the potential of heterogeneously integrated DFB lasers as wavelength transparent flip-flops or high speed signal regenerators.

\section{ACKNOWLEDGMENT}

The authors acknowledge the support of the EU projects HISTORIC and WADIMOS and the Dutch MEMPHIS project. M. Tassaert acknowledges the BOF (Ghent University) for a doctoral grant.

\section{REFERENCES}

[1] R. Van Caenegem, J. A. Martinez, D. Colle, M. Pickavet, P. Demeester, F. Ramos, and J. Marti, "From IP over WDM to all-optical packet switching: conomical view," Journ. Lightw. Techn., Vol. 24, 1638-1645 (2006).

[2] H. J. S. Dorren, M. T. Hill, Y. Liu, N. Calabretta, A. Srivatsa, F. M. Huijskens, H. de Waardt, and G. D. Khoe, "Optical packet switching and buffering by using alloptical signal processing methods," Journ. Lightw.Techn., Vol. 21, pp. 2-12, 2003.

[3] S. Keyvaninia, M. Muneeb, S. Stankovic, P.J. Van Velthoven, D. Van Thourhout, G. Roelkens, "Ultra-thin DVS-BCB adhesive bonding of III-V wafers, dies and multiple dies to a patterned silicon-on-insulator substrate", Optical Materials Express, Vol. 3, pp. 35-46, 2013

[4] J. Van Campenhout, P. Rojo Romeo, P. Regreny, C. Seassal, D. Van Thourhout, S. Verstuyft, L. Di Cioccio, J.M. Fedeli, C. Lagahe, R. Baets, "Electrically pumped InPbased microdisk lasers integrated with a nanophotonic silicon-on-insulator waveguide circuit”, Opt. Express, Vol. 15, pp. 6744-6749, 2007.

[5] L. Liu, R. Kumar, K. Huybrechts, T. Spuessens, G. Roelkens, E-J. Geluk, T. de Vries, P. Regreny, D. Van Thourhout, R. Baets, G. Morthier, "An ultra-small, lowpower all-optical flip-flop memory on a silicon chip", Nature Photonics, Vol. 4, pp. 182-187, 2010.

[6] J. Hofrichter, T. Morf, A. La Porta , O. Raz, H. J. S. Dorren, B. J. Offrein, "A single InP-on-SOI microdisk for high-speed half-duplex on-chip optical links", Opt. Express, Vol. 20, pp. B365-B370, 2012.

[7] M. Tassaert, G. Roelkens, H.J.S. Dorren, D. Van Thourhout, O. Raz, "Bias-free, low power and optically driven membrane InP switch on SOI for remotely configurable photonic packet switches", Opt. Express, Vol. 19, B817-B824, 2011. 